\title{
Globicatella sanguinis: REPORTE DE UN CASO DE MENINGITIS EN EL HOSPITAL MILITAR CENTRAL
}

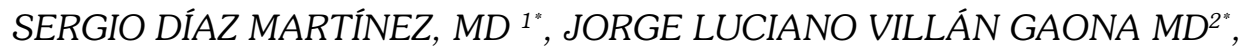 \\ SAMUEL VEGA HURTADO MD ${ }^{3}$, DIANA PATRICIA PACHÓN BARINAS MsC ${ }^{4 *}$, \\ ALBERTO FABIO CABALLERO RESTREPO MD ${ }^{5}$ \\ ${ }^{1}$ Neurocirujano del Hospital de la Misericordia y de la Clínica la Colina. Bogotá-Colombia \\ ${ }^{2}$ Médico-Cirujano del Hospital Profesor Jorge Cavelier. Cajica (Cund)-Colombia \\ ${ }^{3}$ Médico-Cirujano Dirección de Sanidad Ejército Nacional, Colombia \\ ${ }^{4}$ Docente Asociada Facultad de Medicina, Universidad Militar Nueva Granada. Bogotá-Colombia \\ ${ }^{5}$ Neurocirujano Endovascular del Hospital Universitario La Samaritana y de la Clínica Palermo,Bogotá \\ -Colombia \\ *Correspondencia, lucianovillangaona@gmail.com,
}

Recibido: 6 Julio 2016 Aceptado: 3 Febrero 2017

\begin{abstract}
RESUMEN
Globicatella sanguinis es un microorganismo patógeno poco común, coco Gram positivo y catalasa negativo, aunque se aísla en ganado ovino, ha sido descrito como agente infeccioso causante de meningitis en seres humanos. La primera descripción de este patógeno fue realizada por Collins y colaboradores en el año de 1992, se debe resaltar, que antes de este suceso, parte de los casos reportados como meningitis por $S$. viridans, pudieron ser causados en realidad por G. sanguinis debido a la falta de identificación de este ultimo y a la gran similitud en cuanto a morfología y resultados de pruebas bioquímicas. Al respecto, desde la descripción original hace 20 años, la información disponible de G. sanguinis es escasa. Se ha presentado un caso de meningitis asociada a G. sanguinis posterior a un procedimiento quirúrgico en un paciente de 1 año de edad en el Hospital Militar Central.
\end{abstract}

Palabras claves: Globicatella sanguinis; meningitis bacteriana; bacteria Gram positivo; procedimiento quirúrgico. 


\title{
Globicatella sanguinis: REPORT OF A CASE OF MENINGITIS IN THE CENTRAL MILITARY HOSPITAL
}

\begin{abstract}
Globicatella sanguinis is a rare pathogenic microorganism Gram-positive cocci and catalase negative, which although is isolated in sheep, has been described as infectious agent causing meningitis in humans. The first description of this pathogen was made by Colins et al. in 1992, it's necessary to outline that before this event, part of the cases reported as a meningitis cause by $S$. viridans could actually be caused by $G$. sanguinis due to the lack of identification of these ones and the great similarity in terms of morphology and results of biochemical tests. However, after 20 years of the original description, the available information of $G$. sanguinis is limited. A case of meningitis associated with G. sanguinis following a surgical procedure has been reported in a 1-year-old patient at the Central Military Hospital.
\end{abstract}

Key words: Globicatella sanguinis; Meningitis; Gram positive bacteria; surgical procedures

\section{Globicatella sanguinis: \\ RELATÓRIO DE UM CASO DE MENINGITIS NO HOSPITAL MILITAR CENTRAL}

\begin{abstract}
RESUMO
Globicatella sanguinis é um microrganismo patogênico raro, cocos grampositivos e catalase negativos, que embora sejam isolados em ovelhas, foram descritos como agentes infecciosos que causam meningite em seres humanos. A primeira descrição desse patógeno foi feita por Colins et al. Em 1992, é necessário esboçar que, antes deste evento, parte dos casos relatados como causa de meningitis por $S$. viridans poderia ser causada por G. sanguinis devido à falta de identificação desses e à grande semelhança em termos de morfologia $E$ resultados de testes bioquímicos. No entanto, após 20 anos da descrição original, a informação disponível de G. sanguinis é limitada. Um caso de meningite associado a $G$. sanguinis após um procedimento cirúrgico foi relatado em um paciente de 1 ano de idade no Hospital Militar Central.
\end{abstract}

Palavras-chave: Globicatella sanguinis; Meningitis, Bactérias Gram positivas; Procedimentos cirúrgicos. 


\section{Introducción}

En los primeros años de la década de los noventa se describió por primera vez Globicatella sanguinis, microorganismo Gram negativo, que hace parte de la flora normal residente en superficies mucosas de mamíferos incluido el hombre (1). Pese a dicho comportamiento tipo comensal, ha sido aislado en casos de neuroinfecciones, septicemia e infección de vías urinarias (2-5), identificado como un agente capaz de causar un deterioro clínico importante e incluso la muerte $(2,6)$. El presente artículo pretende otorgar una breve descripción acerca de $G$. sanguinis, así como las características de la meningitis asociada a este patógeno, en relación con un procedimiento quirúrgico (tercer ventriculostomia endoscópica), adicionalmente este es el primer caso reportado de $G$. sanuinis asociado a meningitis en Colombia. Para la estructura metodológica en este reporte de caso se siguieron las guías del Desarrollo de Guías Clínicas Basadas en el Consenso por sus siglas en inglés CARE, Tabla 1.

Tabla 1. Lista de chequeo 2016: información a considerar cuando se escribe un reporte de caso, tabla tomada de: The UK EQUATOR Centre is hosted by the Centre for Statistics in Medicine, NDORMS, University of Oxford, disponible en el link http://www.equator-network.org/about-us/uk-equator-centre/

\begin{tabular}{|c|c|c|}
\hline Topic & Item & Checklist item description \\
\hline Title & $\mathbf{1}$ & The words "case report" should be in the title along with the area of focus \\
\hline Key words & 2 & Four to seven key words-including "case report" as a key word \\
\hline Abstract & & $\begin{array}{l}\text { Background: What does this case report add to the medical literature } \\
\text { Case summary (1 paragraph): chief complaint, diagnoses, interventions, and outcomes } \\
\text { Conclusion: What are the main "take-away" lessons from this case? }\end{array}$ \\
\hline Introduction & 4 & How does this case informs healthcare delivery-with references (1-2 paragraphs) \\
\hline Timeline & 5 & Relevant information from this case report organized into a timeline (table or figure) \\
\hline Patient Information & $\begin{array}{l}\mathbf{6 a} \\
\mathbf{6 b} \\
\mathbf{6 c}\end{array}$ & $\begin{array}{l}\text { De-identified demographic and other patient specific information } \\
\text { Chief complaint (what prompted this patient visit) } \\
\text { Relevant medical and psychosocial history (including interventions and outcomes) }\end{array}$ \\
\hline Physical Exam & 7 & Relevant physical examination findings \\
\hline $\begin{array}{l}\text { Diagnostic } \\
\text { Assessment }\end{array}$ & $\begin{array}{l}\mathbf{8 a} \\
\mathbf{8 b} \\
\mathbf{8 c} \\
\mathbf{8 d}\end{array}$ & $\begin{array}{l}\text { Diagnostic evaluations (such as laboratory testing, imaging, surveys) } \\
\text { Diagnoses (consider tables/figures linking assessment with diagnoses and interventions } \\
\text { Diagnostic reasoning including other diagnoses considered and diagnostic challenges } \\
\text { Prognostic characteristics (such as staging in oncology) were applicable }\end{array}$ \\
\hline Interventions & $\begin{array}{l}9 \mathbf{a} \\
\mathbf{9 b} \\
\mathbf{9 c} \\
\mathbf{9 d}\end{array}$ & $\begin{array}{l}\text { Types of intervention (such as pharmacologic, surgical, preventive, self-care) } \\
\text { Intervention administration (such as dosage, strength, duration) } \\
\text { Changes in intervention (whit rationale) } \\
\text { Other concurrent interventions }\end{array}$ \\
\hline $\begin{array}{l}\text { Follow up and Out- } \\
\text { comes }\end{array}$ & $\begin{array}{l}10 a \\
10 b \\
10 \mathbf{c} \\
10 d\end{array}$ & $\begin{array}{l}\text { Clinician and patient-assessed outcomes (when appropriate) } \\
\text { Important follow up diagnosis evaluations } \\
\text { Assessment of intervention adherence and tolerability } \\
\text { Adverse and unanticipated events }\end{array}$ \\
\hline Discussion & $1 \mathbf{1 1 a}$ & $\begin{array}{l}\text { Strengths and limitations in your approach to this case-with references } \\
\text { Conclusion and rationale (including possible causes for outcomes) }\end{array}$ \\
\hline Patient Perspective & 12 & When appropriate include the patient's perspective on this episode of care \\
\hline Informed Consent & 13 & $\begin{array}{l}\text { Patient informed consent is likely to be required by a journal (or your institution) prior to } \\
\text { publication }\end{array}$ \\
\hline Other & 14 & $\begin{array}{l}\text { IRB approval as indicated or needed; Acknowledgement section; Competing interests; } \\
\text { Funding }\end{array}$ \\
\hline
\end{tabular}




\section{Caso Clínico}

Paciente femenina de un año, producto de primera gestación, con adecuada red de apoyo, asiste a consulta por presentar aumento progresivo del perímetro cefálico hace seis meses. Al interrogatorio padres niegan síntomas de hipertensión endocraneana. Examen físico con talla y peso adecuado para la edad, destaca perímetro cefálico $52 \mathrm{~cm}$, superior a percentil 97 según patrón de crecimiento cefálico para niñas entre cero y dos años de la OMS; dados estos hallazgos se realizan imágenes cerebrales (tomografía axial computada de cráneo simple) donde se evidencia estenosis del acueducto de Silvio (Figura 1), razón por la cual se decide manejo quirúrgico con tercer ventriculostomía endoscópica.

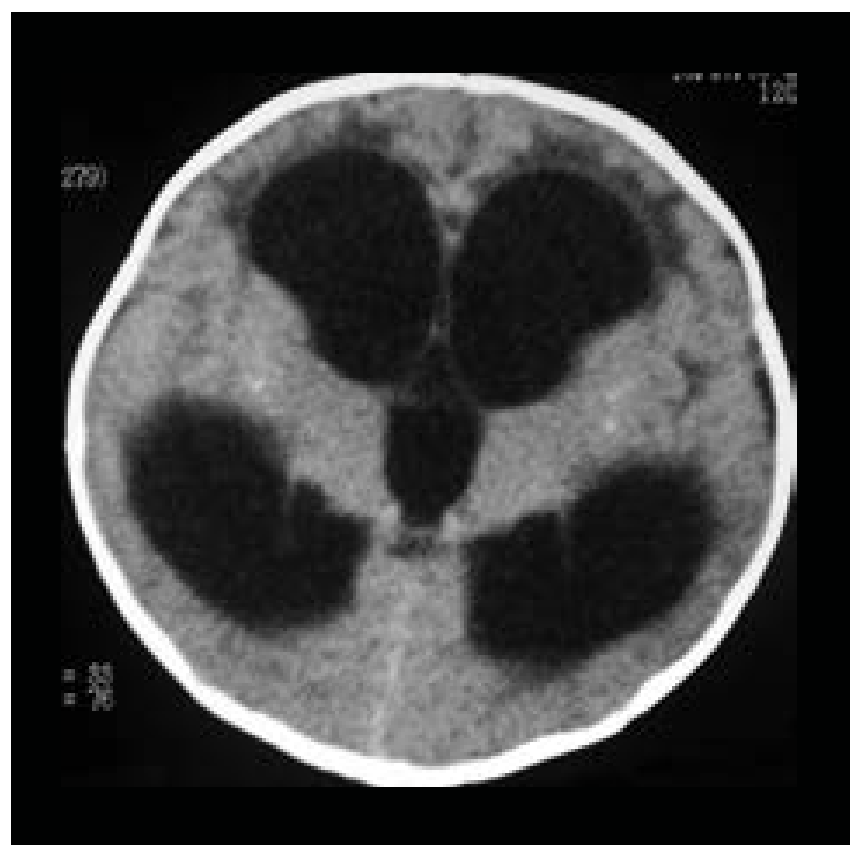

Figura 1. Tomografía Axial Computarizada donde se observa dilatación triventricular sugestiva de estenosis de acueducto de Silvio, imagen de la paciente.

La paciente es llevada a cirugía para procedimiento derivativo endoscópico con neuroendoscopio rígido realizando fenestración de piso de tercer ventrículo, observando adecuada permeabilidad posterior a procedimiento. Tres días posteriores a este procedimiento es dada de alta por presentar adecuada evolución. Paciente reingresa a servicio de urgencias pediátricas pasados 6 días del procedimiento, por cuadro clínico de emesis, irritabilidad, fiebre cuantificada en $38{ }^{\circ} \mathrm{C}$; al examen físico de ingreso, sin alteración de estado de conciencia, destacaba abom- bamiento de fontanela anterior. Paraclínicos de ingreso dentro reportaron de Leucocitos 16.600 con neutrófilos de $90 \%$. Por antecedente reciente de neuro-intervención asociado a hallazgos clínicos y leucocitosis con neutrofilia se sospecha neuroinfección, razón por la cual se realiza punción transfontanelar para estudio de líquido cefalorraquídeo (LCR), el cual presento un aspecto turbio, glucorraquia $74 \mathrm{mg} / \mathrm{dl}$, proteinorraquia 144.7 $\mathrm{mg} / \mathrm{dl}$, Leucorraquia $70 \mathrm{ml} / \mathrm{mm}^{3}$, tinción Gram de dicha muestra (LCR) con presencia de cocos Gram positivos, estudios posteriores indican que el microorganismo es catalasa negativo y produce $\alpha$ hemolísis, razón por la cual se considera necesario realizar prueba de alta especificidad para tipificar el microorganismo en cuestión, por lo que se aplica kit API Strep ${ }^{\circledR}$ encontrándose positivo para Globicatella sanguinis. Luego de estos hallazgos, el servicio de infectopedaitría inicia terapia antibiótica con Vancomicina y Cefepime con el fin de contralar la neuroinfección. La paciente muestra signos de control de la respuesta inflamatoria sistémica tres días después del inicio de antibiticoterapia, también disminución de signos de hipertensión intracraneal. No se encuentran microrganismo en muestras de LCR posteriores al tratamiento con antibióticos, razón por la cual no se realiza nuevo kit API Strep ${ }^{\circledR}$

\section{Discusión}

G. sanguinis es un coco Gram positivo, a hemolítico, catalasa negativa $(4,6-8)$, no móvil, morfología microscópica de forma ovoide, capaz de formar cadenas cortas y colonias puntiformes en medios de cultivo sólidos para microorganismos exigentes (agar chocolate) y como característica destacada en cuanto a su espectro de resistencia muestra sensibilidad a Glicopeptidos (9). Crece a temperaturas entre $10^{\circ} \mathrm{C}$ a $45^{\circ} \mathrm{C}$, en medio anaerobio con agar chocolate enriquecido con $\mathrm{CO}_{2}$ al $5 \%$, bilis esculina, solución salina al $6,5 \%(4,6)$.

Colins y cols. en 1992, identifico el género Globicatella, en base a la secuenciación del gen que codifica la subunidad 16S ribosomal (16S RNAr) de Streptococcus spp (Viridans) $(5,7)$. Estos microorganismos fueron sometidos a un proceso de fenotipificación y filogenia, luego del cual se encontró secuencias genómicas con diferencias significativas propias de un nuevo género, "Globicatella", que contaba con una única especie "G.sanguis" $(4,7)$ renombrada " $G$. sanguinis". Posteriormente se descubrió otra especie "G. sulfidifaciens" (4). Ambas especies se han reportado como agentes infecciosos de animales y en seres humanos, asilándose en pacientes con bacteremia, infecciones del tracto urinario y del sistema 
nervioso central en seres humanos $(2,4,9)$. Sin embargo, se ha demostrado que $G$. sanguinis puede ser parte de la flora a nivel del recto y la ingle comportándose como un microorganismo comensal, ya que se ha asilado en pacientes con ausencia de sintomas, lo que sugiere que puede ser un reservorio $(3,8,9)$, documentándose además su capacidad como microorganismo oportunista en pacientes inmunosuprimidos, en quienes produce cuadros de meningoencefalitis e incluso en abscesos cerebrales (2).

Según la literatura, el cuadro clínico secundario a la meningitis por $G$. sanguinis consiste en fiebre, vomito, cefalea, alteración en la marcha, nistagmus, pérdida del apetito, y ataxia afectando a los pacientes en los extremos de la vida (6,9-12). Se evidencia además pleiocitosis a expensas de neutrófilos acompañado de una extracción de líquido cefaloraquideo de características turbias, con proteinoraquia $(8,9)$. En este caso la paciente presentaba la mayoría de los elementos diagnósticos nombrados anteriormente; estos hallazgos no son muy diferentes de otras meningitis y por si mismos no son suficientes para realizar un análisis acertado (11), si a esto se le suma la gran similitud de las pruebas bioquímicas usuales de otros microorganismos (especialmente St. viridans), se encuentra un diagnostico complicado, que amerita pruebas de alta especificidad como Api Strep ${ }^{\circledR}$ (99.9\%) (9).

Algunos de los signos que se obtienen de la neuroinfección, son petequias y congestión en las meninges, evidenciado en la necropsia de corderos infectados (6), sin embargo, en este caso no se pudo observar esta característica.

En cuanto a su perfil de susceptibilidad antibiótica, G. sanguinis es sensible a la Glucopeptidos $(5,6,8,11)$, Penicilinas $(8,11)$ y Amino-penicilinas (11), pero visiblemente resistente a Macrólidos y a Cefalosporinas $(10,11)$. Este espectro de resistencia es el causante de falla terapéutica en la primera línea en el tratamiento de la meningitis bacteriana aguda en paciente inmunocompetentes; en este punto, la diferenciación molecular, el aislamiento del microorganismo y las pruebas de sensibilidad frente a antibióticos son indispensables para dar un diagnóstico certero.

\section{Conclusión}

La mayoría de meningitis encontradas en nuestro medio son ocasionadas por microrganismos usuales a los cuales nos encontramos acostumbrados en su diagnóstico y tratamiento; aunque la frecuencia de encontrar infec- ciones por microrganismos inusuales es baja, debemos estar atentos y considerar otros gérmenes poco comunes como agente etiológico; el oportuno diagnóstico y tratamiento garantiza al paciente una adecuada recuperación y menos secuelas.

\section{Conflictos de interés}

Los autores declaran no tener de manera directa o indirecta, ningún tipo de conflicto de interés financiero, académico o laboral que puedan poner en peligro la validez de este estudio.

\section{Financiación}

Este trabajo se realizó con el apoyo financiero propio de los autores.

\section{Referencias}

1. Matsunami M, Otsuka Y, Ohkusu K, Sogi M, Kitazono H, Hosokawa N. Urosepsis caused by Globicatella sanguinis and Corynebacterium riegelii in an adult: case report and literature review. J Infect Chemother. 2012; 18(4):5524 Disponible en: https://www.ncbi.nlm.nih.gov/pubmed/22080192?report=docsum\&format=text.DOI: 10.1007/s10156-011-0335-x

2. Assefa S., Rezai F., Delman M., Fless K., Litinski M. YP. Globicatella sanguinis meningoencephalitis and acute brain abscesses in an immunocompromised patient. Crit Care Med. 2012. DOI: $10.1097 / 01 . c c m .0000425445 .30400 .29$

3. Valiente c, fontclara l, ferreira $f$ bh. Globicatella sanguinis: un hallazgo poco frecuente.

4. Lau SKP, Woo PCY, Li NKH, Teng JLL, Leung K-W, $\mathrm{Ng} \mathrm{KHL}$, et al. Globicatella bacteraemia identified by $16 \mathrm{~S}$ ribosomal RNA gene sequencing. J Clin Pathol [Internet]. 2006; 59(3):303-7. Available from: http:// www.pubmedcentral.nih.gov/articlerender.fcgi?artid $=1860353 \&$ tool $=$ pmcentrez\&rendertype $=$ abstract. DOI: $10.1136 /$ jcp.2005.028878.

5. Jain et al. - Globicatella sanguinis meningitis: first case report from Asia. J Infect Dev Ctries 2012; 6(7):592-594, disponible en: http://www.jidc.org/index.php/journal/ article/view/22842948/757. DOI: 22842948.

6. Vela AI, Fernández E, Heras A, Paul A, Domínguez L, Collins MD, et al. Meningoencephalitis Associated with Globicatella sanguinis Infection in Lambs. 2000;4254-6. DOI: 11060102.

7. Collins MD1, Aguirre M, Facklam RR, Shallcross J WA. Globicatella sanguis gen.nov., sp.nov., a new gram-pos- 
itive catalase-negative bacterium from human sources. PubMed. 1992;5:433-7.

DOI: $10.1111 / \mathrm{j} .1365-2672.1992 . t b 05000 . x$.

8. Jain N, Mathur P, Misra MC. Globicatella sanguinis meningitis in a post head trauma patient: first case report from Asia. J Infect Dev Ctries [Internet]. 2012;6(7):592-4. DOI: 22842948.

9. Héry-Arnaud G, Doloy A, Ansart S, Le Lay G, Le FlècheMatéos A, Seizeur R, et al. Globicatella sanguinis meningitis associated with human carriage. J Clin Microbiol [Internet]. 2010;48(4):1491-3. DOI: 10.1128/JCM.01299-09.

10. Seegmüller I, van der Linden M, Heeg C, Reinert RR. Glo- bicatella sanguinis is an etiological agent of ventriculoperitoneal shunt-associated meningitis. J Clin Microbiol [Internet]. 2007;45(2):666-7. DOI: 10.1128/JCM.01774-06.

11. Shewmaker PL, Steigerwalt AG, Weyant R, Facklam RR. DNA Relatedness, Phenotypic Characteristics, and Antimicrobial Susceptibilities of Globicatella sanguinis Strains Susceptibilities of Globicatella sanguinis Strains. 2001; DOI: 10.1128/JCM.39.11.4052-4057.2001.

12. Morales A, Alonso L. Epidemiology of the meningitis: A socieconomic visión. Salud Universidad del Norte. Barranquilla (Col.) 2006; 22 (2): 105-120. Scielo. Disponible en:http://www.scielo.org.co/pdf/sun/v22n2/v22n2a06 